\title{
Simulation, Design and Construction of High Voltage DC Power Supply at 15 kV Output Using Voltage Multiplier Circuits
}

\author{
N. Mariun, D. Ismail, K. Anayet, N. Khan and M. Amran \\ School of Electrical System Engineering, Northern Malaysia University College of Engineering \\ 01000 Kangar, Perlis, Malaysia
}

\begin{abstract}
This study describes the details of high voltage DC power supply whose output voltage is 15 $\mathrm{kV}$. In this study, we review the major aspects of the design of voltage multiplier circuits and constructed a prototype power supply based on simulation, design and implementation of the hardware works in the laboratory. Its simulation works done by using EMTDC PSCAD and PSPICE software. Experimental results are presented to verify the simulation results.
\end{abstract}

Key words: DC power supply, voltage doubler circuits, voltage tripler circuits

\section{Introduction}

DC voltages are chiefly used for pure scientific research work and in the industry level the main application of the DC high voltage is in test on cables with a relatively large capacitance, which takes a very large current if it is tested with AC voltages ${ }^{[1]}$. High voltages are primarily produced for insulation testing of high voltage equipment under power frequency AC, DC, switching and lightning impulse voltages. For insulation testing equipment, the voltages are increased up to several million volts but currents are decreased to a few $\mathrm{mA}$ or maximum 1 ampere both for $\mathrm{AC}$ and $\mathrm{DC}$ test sets. In the fields of electrical engineering and applied physics, high voltage DC are required for several applications such as electron microscopes and $\mathrm{x}$-ray units require high DC voltages of the order of 100 $\mathrm{kV}$ or more, electrostatic precipitators, particle accelerators in nuclear physics and so on ${ }^{[2]}$.

The high voltage equipment is required to study the insulation behavior under all conditions, which the apparatus is likely to encounter. Tests are also made with voltages higher than the normal working voltage to determine the factor of the safety over the working conditions and to ensure that the working margin is neither too high nor too low. The conventional forms of high voltage in use can be divided into the following classes: a) Alternating current voltages b) Direct current voltages and c) Transient voltages ${ }^{[3,4]}$.

In this study, the main emphasis has been given up at first stage on the simulation, design and development of the high voltage DC power supply. At the second stage, the DC power supply is constructed based on hardware implementation and it can be used for multiple purposes. At the first stage of this work is to study the voltage multiplier circuits i.e. voltage doubler circuits, voltage tripler circuits and voltage quadrupler circuits and so on and simulate all the circuits. Finally based on Cockcroft-Walton (C-W) voltage multiplier circuits to construct a prototype DC power supply in the laboratory at the output range of $15 \mathrm{kV}$. The conventional technique is used because the designed multiplier circuit is intended to be applied either for impulse generator charging units or for laser excitation. The main components of the DC power supply are rectifier diodes and capacitors. The simplest unregulated power supply consists of three parts namely, the transformer unit, the rectifiers unit and the capacitors unit.

Joseph M. Beck ${ }^{[5]}$, has presented his paper the basic operation of voltage multiplier circuits such as half wave voltage doubler and tripler circuits and discussed guidelines for electronic component selection for diode and capacitor. Spencer and his group ${ }^{[6]}$, have designed the prototype surface mounted CrockroftWalton $(\mathrm{C}-\mathrm{W})$ board and tested for use in a batteryoperated, palm-sized radiation detection device and it took around $1 \mathrm{kV}$ output voltage and current less than $15 \mathrm{~mA}$. In addition to circuit components, this board contains sockets contains which hold two Hamamatsu R7400P PMTs. Juichi Tanaka, et al ${ }^{[7]}$, has explained the new idea to develop the high voltage DC power supply. They introduced a high frequency switching converter, as a result its shape becomes smaller. The conventional $\mathrm{C}-\mathrm{W}$ multiplier circuit ignores the inductance but they have used the inductance as well. They were able to produce $70 \mathrm{kV}, 0.15$-ampere DC power supply. Aintablian, et al ${ }^{[8]}$, have discussed about the single-phase harmonic reduction circuit based on voltage doubler circuit using switch operated on line frequency, instead of using switch mode technology. The advantage of this circuit is that low cost, high reliability and simplicity of control. The disadvantage of this circuit is that it cannot completely control the harmonic current. John C, Salmon ${ }^{[9]}$, has explained single phase voltage doubler PWM boost rectifiers and

Corresponding Author: D. Ismail, K. Anayet, School of Electrical System Engineering, Northern Malaysia University College of Engineering, 01000 Kangar, Perlis, Malaysia. 
obtain three types of PWM voltage switching patterns namely, unipolar PWM, Bipolar PWM, and phase adjusted unipolar PWM. When high output voltage is required, the voltage doubler rectifier is able to generate AC line currents with the lowest current distortion. Yamamoto and his group, ${ }^{[10]}$, have proposed a power factor correction scheme using a voltage doubler rectifier circuit without switching devices. In this method using a voltage doubler rectifier, the input current is divided into two periods, where one period charges the small input capacitor and the other charges the large output capacitor through a filter capacitor.

Zhang, et al ${ }^{[11]}$, in their paper have discussed about the experimental results of the voltage tripler circuit. They proposed improved voltage tripler with a symmetrical stacking charge pump. The main advantage of using this symmetrical staking charge pump is to have a rapid rise time for the output voltage. Zhang, et al ${ }^{[12]}$, in their paper have discussed about the experimental results of the voltage tripler circuit. They proposed improved voltage tripler with a symmetrical stacking charge pump. The main advantage of using this symmetrical staking charge pump is to have a rapid rise time for the output voltage.

Voltage multipliers: It is already known how a transformer functions to increase or decrease voltages. It is also learned that a transformer secondary may provide one or several AC voltage outputs, which may be greater or less than the input voltage. When voltages are stepped up, current is decreased; when voltages are stepped down, current is increased. There is another method for increasing voltages that is known as voltage multiplication. Voltage multipliers are used primarily to develop high voltages where low current is required. The measured output voltage of a voltage multiplier may be several times greater than the input voltage. For this reason, voltage multipliers are used only in special applications where the load is constant and has high impedance or where input voltage stability is not critical. Voltage multipliers may be classified as voltage doubler, tripler or quadrupler. The classification depends on the ratio of the output voltage to the input voltage. For example, a voltage multiplier that increases the peak input voltage twice is called a voltage doubler. The figure used in the explanation of voltage multipliers in this works shows a transformer input, even though for some applications a transformer is not necessary. The input could be directly from the power source or line voltage. This, of course, does not isolate the equipment from the line and creates a potentially hazardous condition. Most military equipments use transformers to minimize this hazard ${ }^{[13]}$.

Voltage doubling principle: Figure 1 shows the schematic for a half-wave voltage doubler ${ }^{[1,2,3,4]}$. In fact, the doubler shown is made up of two half-wave voltage rectifiers. Here $C_{1}, D_{1}$ make up one half-wave rectifier and $C_{2}, D_{2}$ make up the other rectifier.

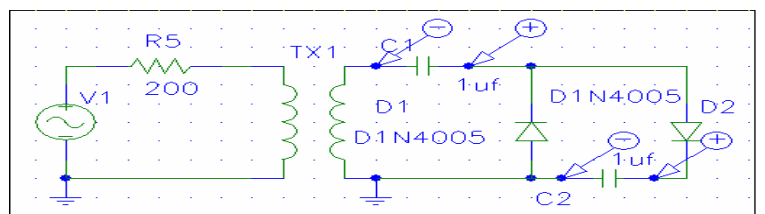

Fig. 1: Connection diagram half-wave voltage doubler

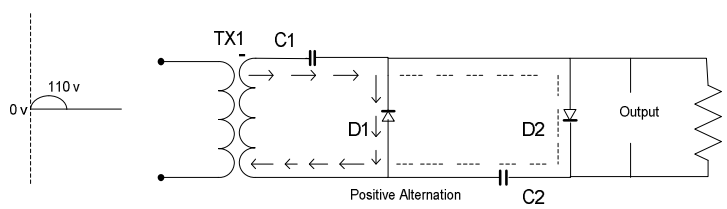

Fig. 2: Voltage doubler in positive alternation

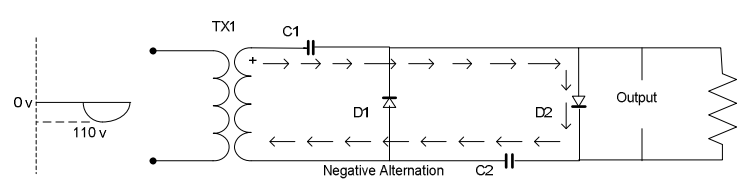

Fig. 3: Voltage doubler in negative alternation

The schematic of the first half-wave rectifier is indicated by the arrow lines in Fig. 2. The dash lines and associated components represent the other halfwave rectifier. Notice that $C_{1}$ and $D_{1}$ work exactly like a half-wave rectifier.

During the positive alternation of the input cycle in Fig. 2, the polarity across the secondary winding of the transformer is as shown. Note that the top of the secondary is negative. At this time $D_{1}$ is forward biased (cathode negative with respect to the anode).

This forward bias causes $D_{1}$ to function like a closed switch i.e. short circuit and allows current to follow the path indicated by the arrows. At this time, $\mathrm{C}_{1}$ charges to the peak value of the input voltage or 220 volts, with the polarity shown for voltage direction. It means voltage direction would be negative to positive direction.

During the period when the input cycle is negative, as shown in Fig. 3, the polarity across the secondary of the transformer is reversed. Note that the top of the secondary winding is now positive.

At this condition now $D_{2}$ is forward biased and $D_{1}$ is reverse biased. A series circuit now exists consisting of $\mathrm{C}_{1}, \mathrm{D}_{2}, \mathrm{C}_{2}$ and the secondary of the transformer. The current flow is indicated by the arrows in Fig. 3. The secondary voltage of the transformer now aids the voltage on $C_{1}$. This results in a pulsating DC voltage of 440 volts. Finally for voltage doubling effect voltage direction would be positive to negative as shown in Fig. $3^{[13]}$.

Voltage tripling principle: The schematic shown in Fig. 4 is an illustration of a half-wave voltage tripler $[1,2,3,4]$. 


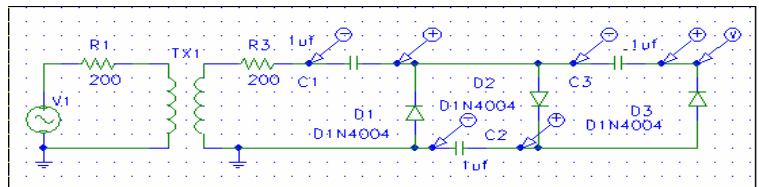

Fig. 4: Connection diagram half-wave voltage tripler.

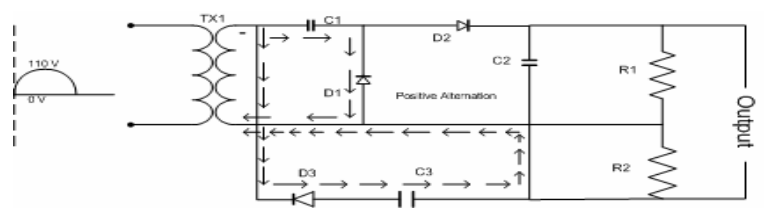

Fig. 5: Voltage tripler in positive alternation

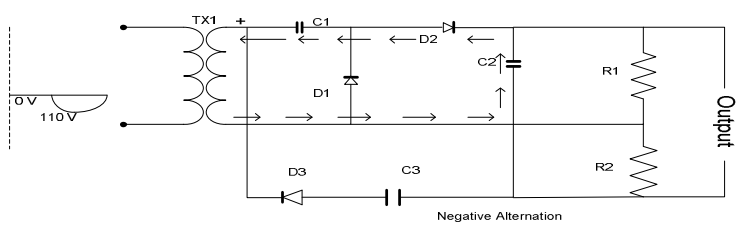

Fig. 6: Voltage tripler in negative alternation

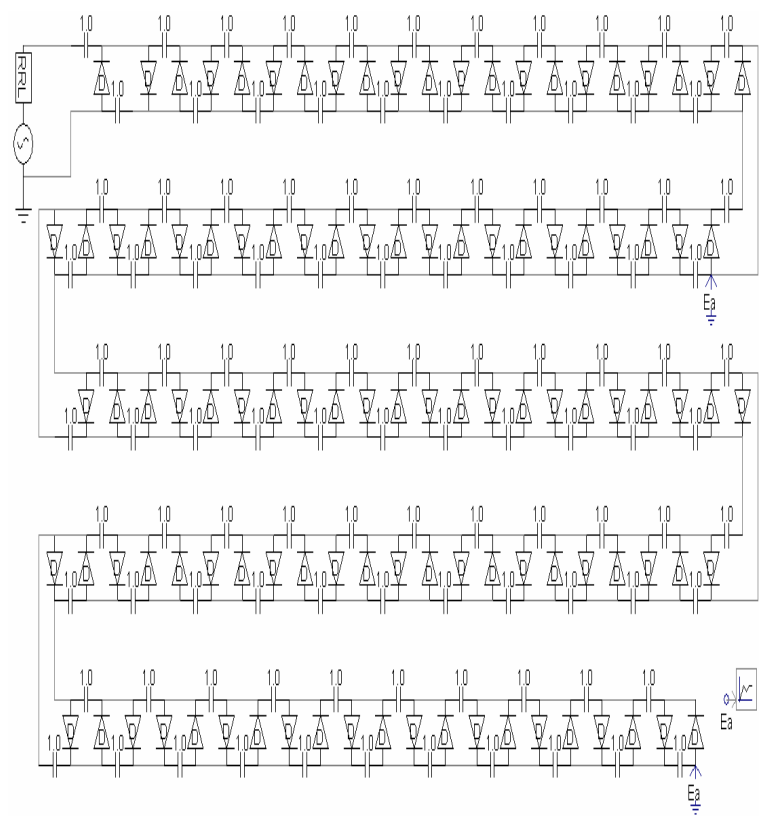

Fig. 7: Proposed $15 \mathrm{kV}$ DC power supply simulation circuit by PSCAD simulation works

In Fig. 5 shows the positive alternation schematic for the voltage tripler. During the period when the input cycle is positive in Fig. 5, the polarity across the secondary winding of the transformer is as shown. Note that the top of the secondary is negative. At this time diode $D_{3}$ is forward biased (cathode negative with respect to the anode) and functions like a closed switch. This allows $\mathrm{C}_{3}$ to charge to a peak voltage of 220 volts and at the same time $C_{1}$ is also charging to 220 volts.

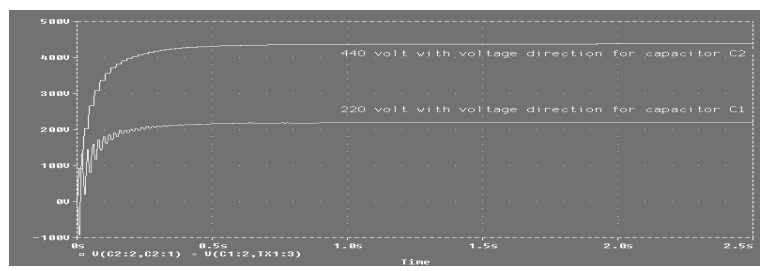

Fig. 8: Simulation result of the voltage doubler circuit by PSpice

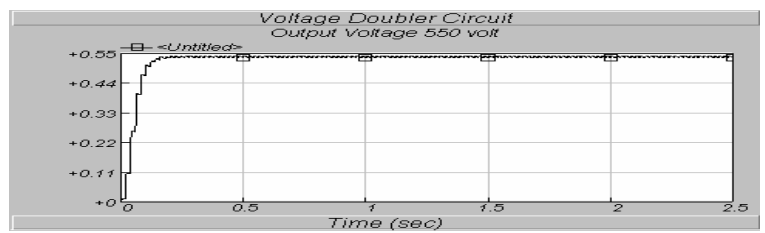

Fig. 9: Simulation result of the voltage doubler circuit by PSCAD

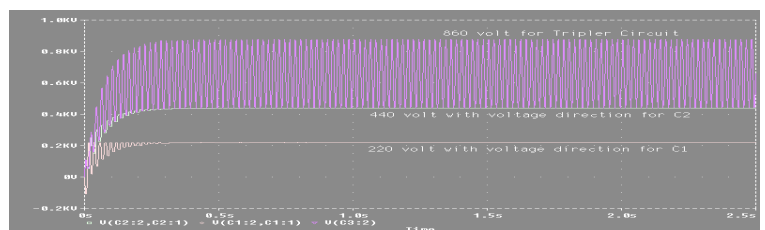

Fig. 10: Simulation result of the voltage tripler circuit by PSpice

Figure 6 shows the period when the input cycle is negative. Here $C_{2}$ is charged to twice the input voltage or 440 volts, as a result of the voltage-doubling action of the transformer and $\mathrm{C}_{1}$. At this time, $\mathrm{C}_{2}$ and $\mathrm{C}_{3}$ are used as series-aiding devices and the output voltage increases to the sum of their respective voltages or 660 volts. $\mathrm{R}_{1}$ and $\mathrm{R}_{2}$ are proportional according to the voltages across $C_{2}$ and $C_{3}{ }^{[13]}$.

The input of the voltage doubler circuit for the simulation has been set to 220 volt and the output obtained is 440 volt. It shows the voltage doubler circuit functioning as expected in Fig. 8.

The input voltage of the voltage doubler circuit for the simulation purpose has been set to 220 volt and the output obtained is 550 volt. It shows that the voltage doubler circuit is functioning as expected and it is shown in Fig. 9. In addition it is noted that the simulation output for PSCAD, it seems to be almost similar with experimental output.

The input voltage of the voltage tripler circuit for the simulation purpose has been set to 220 volt and the output obtained is 860 volt. It shows that the voltage tripler circuit is functioning as expected and it is shown in Fig. 10.

The input voltage of the voltage tripler circuit for the simulation purpose has been set to 220 volt and the output obtained is 1050 volt. It shows that the voltage tripler circuit is functioning as expected and it is shown in Fig. 11. In addition it is noted that the simulation output for PSCAD, it seems to be more than experimental output. 


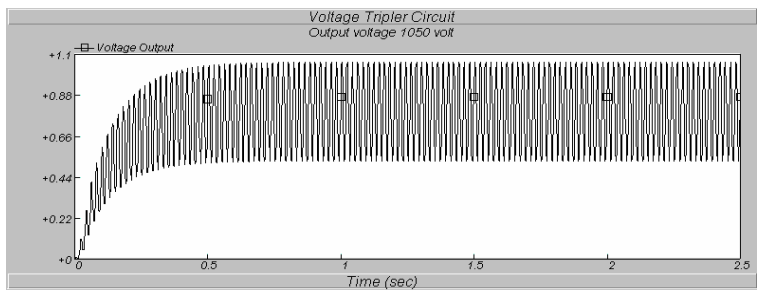

Fig. 11: Simulation result of the voltage tripler circuit by PSCAD

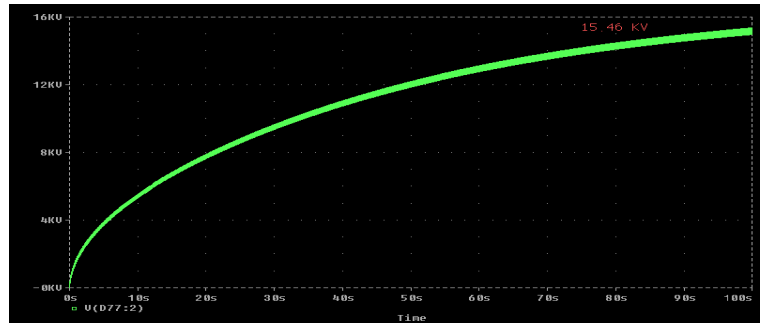

Fig. 12: Simulation output $15 \mathrm{kV}$ DC power supply by PSpice

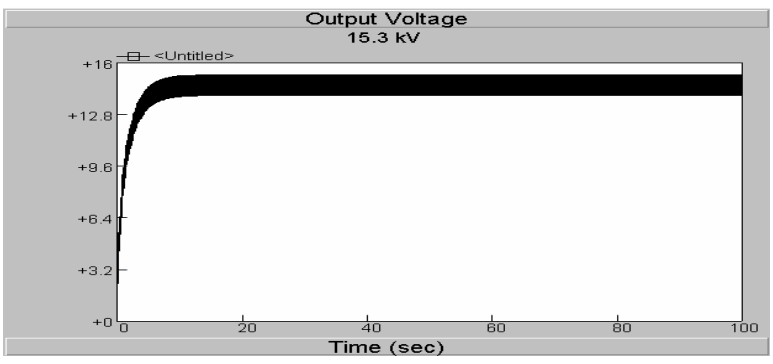

Fig. 13: Simulation output $15 \mathrm{kV}$ DC power supply by PSCAD

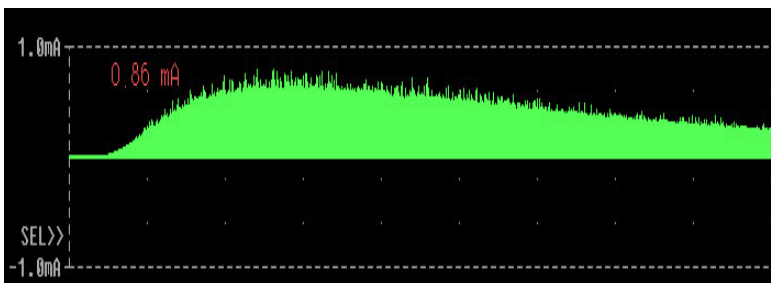

Fig. 14: Simulation output current

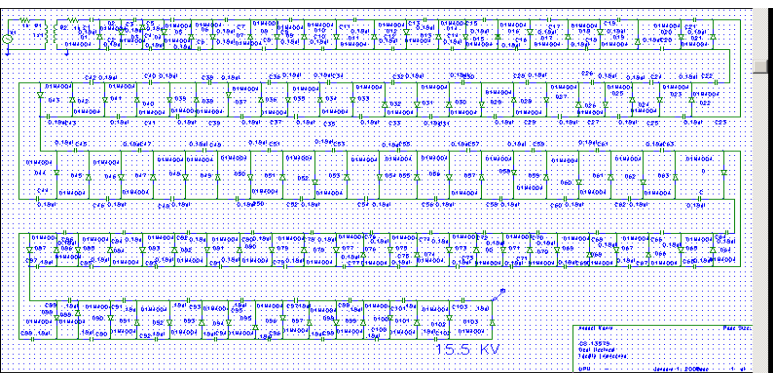

Fig. 15: Proposed 15kV DC power supply simulation circuit by PSpice

Figure 12 and 13 shows the simulation output voltage and Fig. 14 shows the output current of the designed high voltage DC power supply for the proposed $15 \mathrm{kV}$ circuit shown as in Fig. 7 and 15 based on PSpice and PSCAD software.

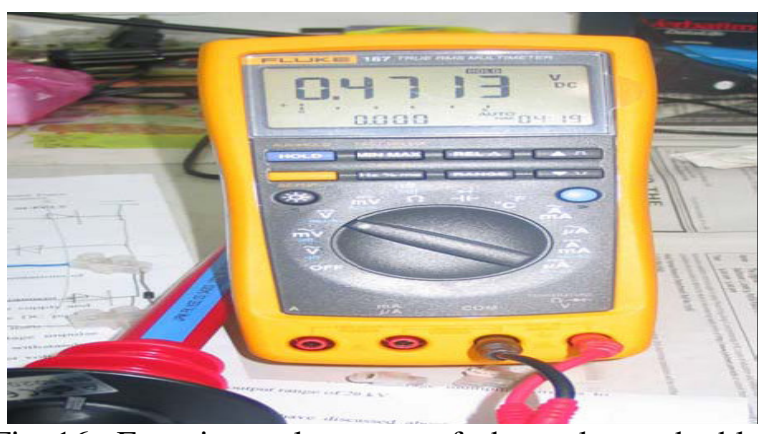

Fig. 16: Experimental output of the voltage doubler circuit

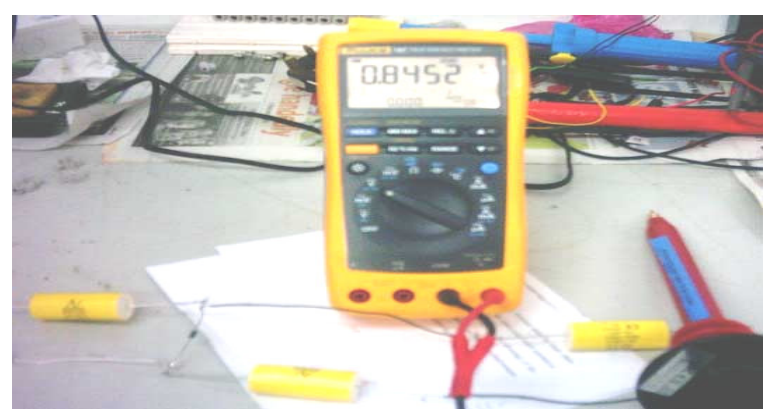

Fig. 17: Experimental output of the voltage tripler circuit

Experimental works: The simulation results are corroborated by implementing and testing the circuits in the laboratory. The input voltage was set at 220 volt and the output obtained is 471 volt for voltage doubler circuit. So according to the simulation circuit, it should be 440 volt but it has given a higher value than its simulation result as shown in Fig. 16.

The simulation results are corroborated by implementing and testing the circuits in the laboratory. The input voltage was set at $220 \mathrm{~V}$ and the output obtained is $845 \mathrm{~V}$ as in Fig. 17. According to the simulation circuit, it should be $860 \mathrm{~V}$ and $1050 \mathrm{~V}$ respectively as in Fig. 10 and 11.

Percentage difference by PSpice $=\frac{(845-860)}{860} \times 100=$ $-1.75 \%$ (negative)

Percentage difference by PSCAD $=\frac{(845-1050)}{1050} \times 100=$ $-19.5 \%$ (negative)

Where 845 volt is the experimental output and expected output is 860 \& $1050 \mathrm{~V}$ for PSpice \& PSCAD respectively.

Figure 16 and 17 is the experimental output voltage of doubler and tripler circuit and Fig. 19 shows that the output of the designed DC power supply is $15 \mathrm{kV}$ and it is tested and installed. 


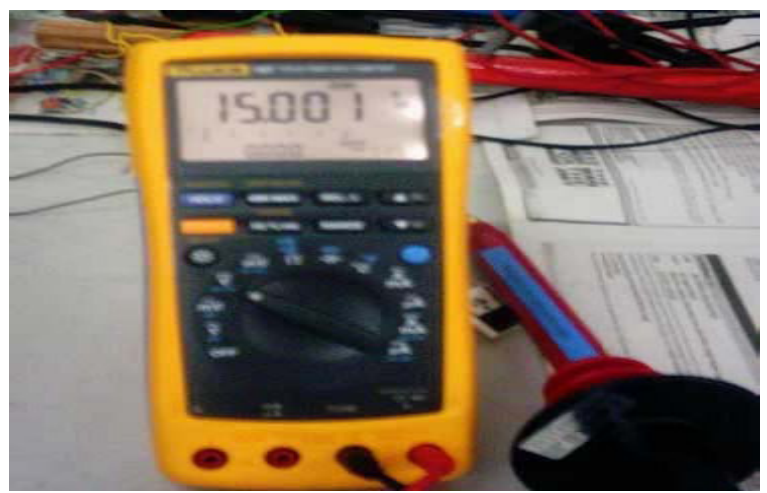

Fig. 18: Output Voltage of DC power supply

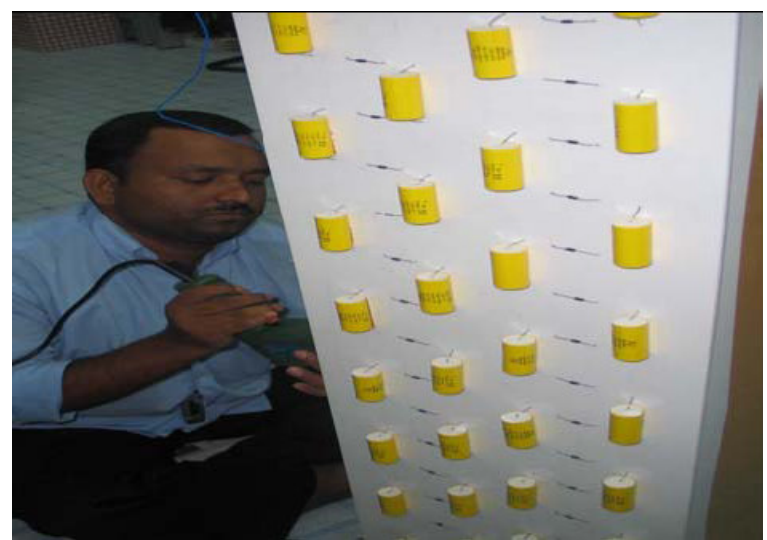

Fig. 19: Constructed DC power supply

Recommendation for future work: After developing the DC power supply at $15 \mathrm{kV}$ output and handling all the simulation and hardware/experimental results and overall situation, it is noted that the developed DC power supply based on $\mathrm{C}-\mathrm{W}$ voltage multiplier circuit, is a unique designed and developed for a special application.

Developed power supply, it is unique because voltage multiplier circuits are diode circuits and they have the advantages of being simple solid state circuits with fairly low parts count and being able to produce output voltages much higher than the input voltage according to project demand. Most DC test sets are done at lower frequency instead of high frequency. It is less expensive, less insulation is required and its range can start from even $1 \mathrm{kV}$ instead of MV capacity compare to the other methods ${ }^{[1,2,3,4]}$.

The fabricated DC power supply, it is a single pulse voltage multiplier since it has only one rectified wave or pulse per cycle. Hence its ripple content is high. In order to reduce the ripple as well as regulation and to improve the efficiency and power output, it is recommended to continue two or more pulse units per cycle ${ }^{[2]}$. Another recommendation is that based on CW multiplier circuits, the designed DC power supply structure is not compact, nowadays the trends to make it smaller in shape, if one can follow the high frequency switching converter technology, then its shape will be reduced based on consumer demand ${ }^{[7]}$.

\section{Conclusion}

The following conclusions could be made from this work based on the above description.

* From the simulation and hardware implementation it is noted that a PSpice and PSCAD based design for high voltage DC power supply at $15 \mathrm{kV}$ output has been proposed and developed. The system hardware has been successfully implemented and tested in the laboratory. The simulation and experimental results have been observed to be in agreement except due to the tolerances of the capacitor.

* Cascaded voltage multipliers are used when larger output voltages are needed without changing the input transformer voltage level. It is used only in special applications where the load is constant and has high impedance or where input voltage stability is not critical.

* This kind of power supply is simple and less expensive. The initial cost of this project is very high as it was developed for the first time. But for mass production the developing cost will be much reduced because a few expensive items which was associated for the first time development can be eliminated.

* The conventional technique is used because the designed multiplier circuit is intended to be applied either for impulse generator charging units or for laser excitation. The main components of the DC power supply are rectifier diodes and capacitors. The simplest unregulated power supply consists of three parts namely, the transformer unit, the rectifiers unit and the capacitors unit.

\section{Acknowledgements}

This work was supported by University Putra Malaysia, Department of Electrical and Electronic Engineering, Faculty of Engineering, 43300-Sri Serdang, Selangor, Malaysia.

\section{References}

1. Kuffel, E. and M. Abdullah, 1984. High Voltage Engineering. Pergamon Press, Oxford.

2. Naidu, M.S. and V. Kamaraju, 2004. High Voltage Engineering. Third Edn., Mcgraw- Hill Company Ltd.

3. Khan, N., 2004. Lectures on Art and Science of High Voltage Engineering. Published in Pakistan.

4. Mazen, A.S. and R. Radwan, 2000. High Voltage Engineering Theory and Practice. Sec. Edn., Revised and Expanded, Marcel Dekker, Inc. 
5. Joseph, M.B. Using rectifiers in voltage multipliers circuits. General Semiconductor.

6. Spencer, D.F., R. Aryaeinejad and E.L. Reber. Using the Cockroft-Walton Voltage Multiplier Design in Handheld Devices. Idaho National Engineering and Environmental Laboratory, P.O. Box 1625, Idaho Falls, ID 83415.

7. Juichi, T. and I. Yuzurihara, 1988. The high frequency drive of a new multi-stage Rectifier Circuit. Kyosan Electric Mfg. Co. Ltd., 2-29, Heian- Cho, Tsurumi-Ku, Yokohama, 230, Japan, IEEE.

8. Aintablian, H.O. and H.W. Hill, 1994. A new single phase AC to DC harmonic reduction converter based on the voltage -doubler circuit. Department of Electrical and Computer Engineering, Ohio University, Athens, IEEE.

9. John, C.S., 1993. Circuit topologies for single phase voltage -doublers Boost rectifiers. IEEE Trans. Power Electr., 8: 4.
10. Yamamoto, I., K. Matsui and F. Ueda, 2000. A power factor correction with voltage doubler rectifier. Chubu University, Dep. Electrical Eng. Japan, IEEE.

11. Zhang, M., N.L. Iaser and F. Devos. An optimized design of an improved voltage tripler. IEF, AXIS, University of Paris, 91405 Orsay, France.

12. Zhang, M., N. Liaser and F. Devos, 2002. Experimental results of an optimized voltage Tripler. IEF, AXIS, University of Paris, 91405 Orsay, France.

13. http://www.infodotinc.com/neets/book7/27m.htm, Electrical Engineering Training Series- Voltage Multipliers. Integrated Publishing, 26838 I-45 North, PMB 102, Spring, TX 77386, USA. 